\title{
Bilateral Taurodontism: A Case Report
}

\author{
Dr Dayanand Chole ${ }^{1}$, Dr Sumit Tambake ${ }^{2}$, Dr Shashank Kundoor ${ }^{3}$, \\ Dr Srinivas Bakle ${ }^{4}$, Dr Neha Gandhi ${ }^{5}$, Dr Rucha Deshpande ${ }^{6}$ \\ ${ }^{1}$ Professor and Head: Department of Conservative dentistry and Endodontics, P.D.U Dental College, Solapur \\ ${ }^{2}$ Post Graduate Student: Department Of Conservative Dentistry and Endodontics, PDU Dental College, Solapur \\ ${ }^{3,4}$ Reader: Department Of Conservative Dentistry and Endodontics, PDU Dental College, Solapur \\ ${ }^{5,6}$ Senior Lecturer: Department Of Conservative Dentistry and Endodontics, PDU Dental College, Solapur
}

\begin{abstract}
Introduction: Taurodontism is a morphologic variation which is characterized by wide elongated pulp chamber, apically displaced pulpal floor and short roots.

Objective: To describe the case of bilateral taurodontism in a female patient with no other associated syndrome.

Case Report: An 55 years old female was referred to the postgraduate endodontic department. The patient's medical history was noncontributory. On intraoral examination showed proximal caries with 36 and was positive to percussion. History of access opening with the same tooth. Radiographic examination showed the characteristics of taurodontism and symptomatic apical periodontitis. The tooth was endodontically treated in 2 appointments. Radiograph of contralateral tooth 46 showed features of taurodontism suggesting case of bilateral taurodontism.

Conclusion: The present case describes bilateral taurodontism with no specific syndromes and endodontic treatment of a hypertaurodont mandibular first molar. Magnification using dental operating microscope is very useful in the evaluation and management of the anatomic complexity of roots and root canals of taurodonts.

Key Words: Bilateral, morphologic variation, taurodontism, pulp chamber.
\end{abstract}

\section{Introduction}

Taurodontism is a morphologic variation generally occurring in multirooted teeth, which is characterized by wide enlarged, elongated pulp chamber with the apical displacement of pulpal floor. ${ }^{1}$ On the other hand, it lacks the cervical constriction which is characteristic of this area, and the roots are extremely short.

Witkop defined "Taurodontism as teeth with large pulp chamber in which bifurcation and trifurcation are displaced apically, so that chamber has greater apico-occlusal height than in normal teeth and lacks the constriction at the level of cemento-enamel junction(CEJ)".

It may be found unilaterally or bilaterally, with no sex predilection. It affects both permanent and deciduous teeth; The former are the most affected, involving one or several teeth in the same quadrant. ${ }^{3}$ Taurodontism was first repoted by Gorjanovic-Kramberger in 1908. However, the term was introduced by Sir Arthur Keith in 1913. He described the Latin "tauro" means bull and Greek "odonto" means tooth (i.e. bull tooth). ${ }^{4}$

Its etiology is still unknown, but it has been suggested that it may be caused by a failure of invagination of diaphragm of Hertwig's epithelial root sheath at the adequate time and horizontal level. ${ }^{5}$ It is also known to be associated with genetic or developmental disorders such as Down syndrome, Klinefelter syndrome, Ectodermal dysplasia, Smith-Magenis syndrome, Thalassaemia major, Tricho-dento-osseous syndrome, Cleft lip and palate and Vander Woude syndrome. Numerous studies suggest that multiple taurodontism is linked to systemic syndromes, whereas a few conflicting findings have been also reported. ${ }^{6}$

Taurodonts can be further classified into Hypotaurodonts, Mesotaurodonts, and Hypertaurodonts according to the severity of taurodontism. ${ }^{7}$ Later, a quantitative expedient to identify taurodontism from radiographs was suggested by Shifman and Chanannel. ${ }^{1}$ They proposed a taurodontic index (TI) obtained by dividing the height of the pulp chamber by the distance between the occlusal end of the pulp chamber and the root apex and then multiplying the number by 100 . A tooth is diagnosed with taurodontism if the index (TI) is equal to or greater than 20 . 


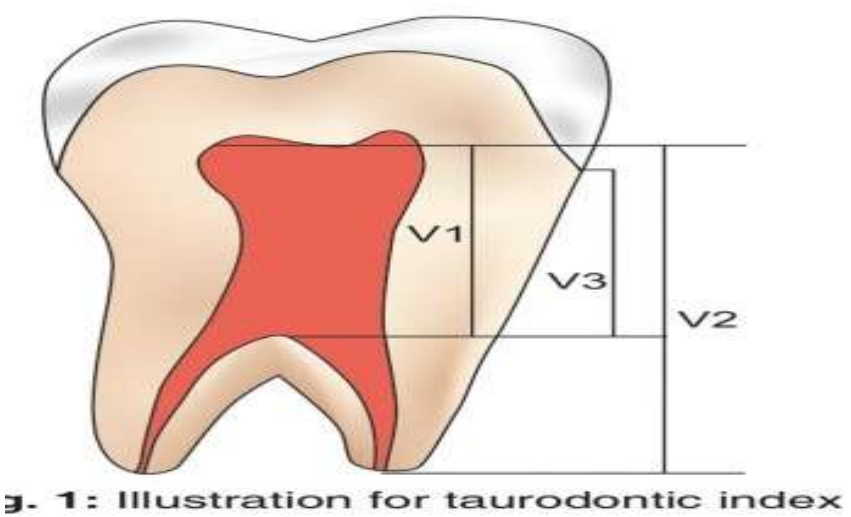

FIG 1 Taurodontism index: vertical height of the pulp chamber (V1), distance between the lowest point of the roof of the pulp chamber to the apex of the longest root (V2), and distance between the baseline connecting the two CEJ and the highest point in the floor of the pulp chamber (V3).

Establishing a condition of taurodontism is made when V1 is divided by V2 and multiplied by 100 if above 20, and V3 exceeds $2.5 \mathrm{~mm}$ : (V1/V2) * $100 \_20$ and V3 _ $2.5 \mathrm{~mm}$. Taurodontic index (TI) _ V1/V2 _ 100. Taurodontismis diagnosed in molars in which TI is above 20 and variable 3 exceeds $2.5 \mathrm{~mm}$.

Degrees of taurodontism were determined as: hypotaurodontism: TI 20-30, mesotaurodontism: TI 3040, and hypertaurodontism: TI 40-75.

In this case, TI equaled 43.56 and V3 5mm clearly indicating hypertaurodontism. The objective of this case report was to describe the characteristics of bilateral multiple taurodontism in a patient with no apparent association to any syndrome.

\section{Case Report}

An 55-year-old female patient was referred to the postgraduate department of Conservative Dentistry and Endodontics. Patient was referred because of inability to find the root canals.

The patient's chief complaint was a spontaneous, sharp pain from the mandibular left quadrant. Her medical history was noncontributory. No significant signs of pathosis were found during the extraoral examination. The intraoral examination revealed that tooth 36 had extensive occlusal destruction and history of access opening.

Dentist had done the access opening with the same tooth, tooth was positive to percussion. Radiographic examination showed the characteristics consistent with taurodontism including an oversized pulp chamber and short roots with 36 (figure 1). To confirm this a radiograph of contralateral tooth was taken, it was also taurodont 46 (figure 2). The pulpal and periradicular diagnosis for tooth 36 was symptomatic apical periodontitis.

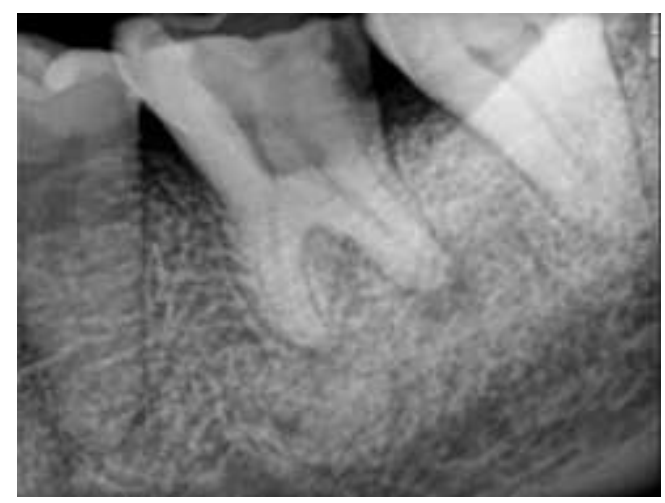

(figure.1 preoperative radiograph)

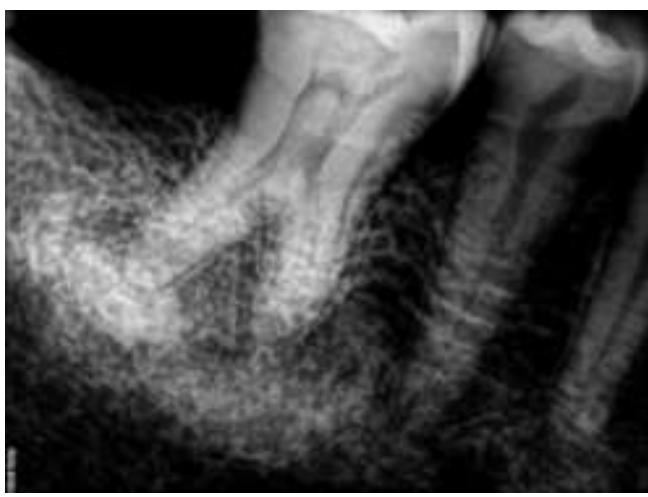

(figure.2 contralateral tooth)

After informed consent was obtained from the patient, tooth 36 was anesthetized with $2 \%$ lidocaine (1:100,000 epinephrine) and isolated with a rubber dam. All the subsequent procedures were performed under a surgical microscope. Occlusal caries were removed with a high-speed round bur. An access cavity was refined, and pulp tissues were removed in the pulp chamber with copious irrigation with $2.5 \%$ sodium hypochlorite solution. As there was calcification in the pulp chamber, was removed using ultrasonics. Three canals 
(mesiobuccal, mesiolingual, and distal canals) were negotiated, which was about $14 \mathrm{~mm}$ from the occlusal surface. Working lengths were determined ( $17 \mathrm{~mm}$ ) using apex locator (Root ZX II; J. Morita ) and confirmed radiographically (figure.3). The canals were cleaned and shaped up to size 25 no $4 \%$ for mesiobuccal and distobuccal, for distal $304 \%$ using (Heroshaper rotary files) NiTi files and 2.5\% sodium hypochlorite and normal saline solution was used for irrigation. The canals were dried with sterile paper points, and calcium hydroxide was placed as a intracanal medicament. The tooth was temporized with Cavit (3MESPE, St Paul, MN). Patient was recalled after 15 days.

In next appointment, calcium hydroxide was removed using $17 \%$ EDTA, canals were irrigated using normal saline. Master cone was selected which was confirmed by radiograph (figure 4). Obturation was done using single cone technique and vertical compaction near canal orifice using Guttapercha and sealapex sealer. Post endodontic restoration was done and patient referred to department of prosthodontics for definite restorative treatment (figure 5).

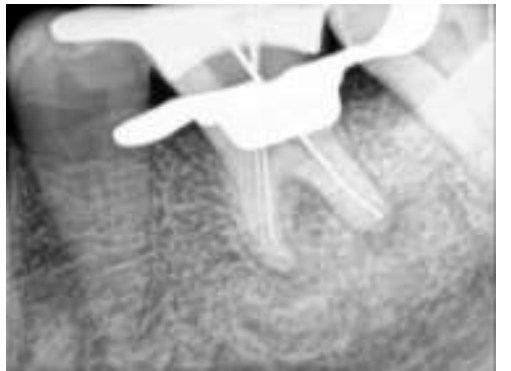

fig.3 working length radiograph

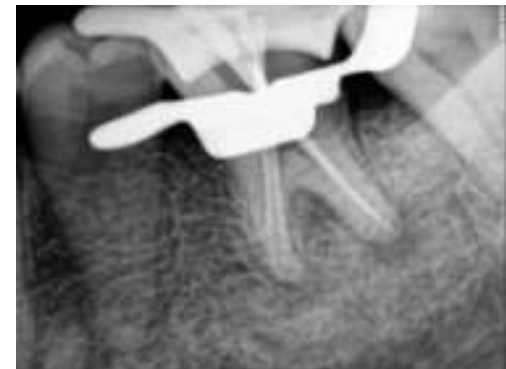

fig.4 master cone radiograph

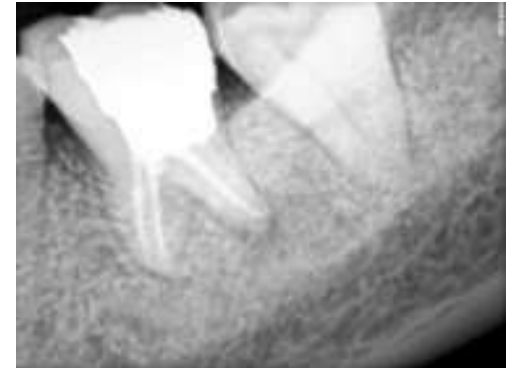

fig.5 post endodontic restoration

\section{Discussion}

Taurodontism is frequently associated with other anomalies and syndromes. ${ }^{6}$ In this case, the patient was a healthy female with no known diseases. Endodontic treatment in taurodont teeth has been described as complex and difficult due to its elongated pulp chamber and apically displaced canal orifices which are difficult to locate without magnification. In such teeth success endodontic treatment largely depends upon use of dental microscope. Use of magnification enhances the visualization of pulpal floor by better illumination of depth of operating field. Durr et al. suggested that morphology could hamper the location of the orifices, thus creating difficulty in instrumentation and obturation. ${ }^{4}$ Two of five cases presented by Cohen and Taintor required endodontic therapy, in which treatment was difficult to complete. ${ }^{4}$ The number of root canals varied in each case . Hayashi reported complicated endodontic treatment of a mandibular molar with taurodontism, where five canals were found, but only three could be instrumented to the apex. ${ }^{4}$

Because of the highly variable morphology of taurodonts, nonsurgical root canal therapy in these teeth is a challenge to clinicians. Elongated pulp chambers and various root canal shapes in taurodonts may make it more difficult for clinicians to locate, instrument, and obturate root canals. ${ }^{8,9,10-12}$ Excessive bleeding observed during the access may also confuse clinicians with perforations because of a greater volume of pulp tissues in the pulp chambers. A high concentration of sodium hypochlorite can be recommended to dissolve the pulp tissue efficiently before the orifices of canals are to be identified. In the present case, pulp chamber was irrigated with $2.5 \%$ sodium hypochlorite during the access preparation of tooth. ${ }^{4}$ Hypertaurodonts often have a thin cervical tooth structure near the canal orifices. Therefore, great care was taken not to remove excessive coronal dentin when canals were located in tooth. Cervical dentin near the canal orifices should be minimally removed under high magnification when a straight-line access is to be obtained, especially in hypertaurodonts.

As the canal was calicified and to save the radicular dentin $4 \%$ taper NiTi file was used to shape the canals. Obturation was done using single cone technique with vertical compaction near the canal orifice.

The present case describes successful completion of endodontic treatment of a taurodont mandibular first molar, which seemed impossible to perform with conventional techniques. Success was mostly attributed to the use of magnification, which easily showed the location of the three canal orifices. Unfortunately, the patient was unavailable for future follow ups. To the best of our knowledge, no long-term follow up studies have been published regarding taurodont endodontically treated teeth.

\section{Conclusion}

Taurodont teeth show wide variations in the size and shape of pulp chambers, varying degrees of obliteration and canal complexity, low canal orifices, and the potential for additional root canal systems.

In performing root canal treatment on these teeth, one should appreciate the complexity of the root canal system. Careful exploration of the grooves between all orifices, particularly with magnification and ultrasonic irrigation. 


\section{References}

[1] Igor Tsesis, Arie Shifman, and Arieh Y. Kaufman. Taurodontism: an Endodontic Challenge. Report of a Case.The American Association of Endodontists VOL. 29, NO. 5, MAY 2003.

[2] Dakshita Joy Waghela, Ashish Amit Sinha. Taurodontism of multiple teeth: a case report Lond Engl 2012.

[3] Sebastian Burklein. Endodontic treatment of two pyramidal (taurodont) mandibular molars : a case report Lond Engl 2008.

[4] H. Jafarzadeh, A. Azarpazhooh , J. T. Mayhall. Taurodontism: a review of the condition and endodontic treatment challenges. International Endodontic Journal, 41, 375-388, 2008.

[5] Amr Radwan, Sahng G. Kim. Treatment of a Hypertaurodontic Maxillary Second Molar in a Patient with 10 Taurodonts: A Case Report.JOE - Vol 40, Number 1, January 2014.

[6] Amrita Jaya Krishan, Dileep Thomas, Akhter Husain. Clinical images in Oral Medicine and Maxillofacial Radiology. Quintessence International 2001

[7] Neira Trinidad Chaparro Gonza'lez, Jesu’s Sebastia’n Leidenz Bermudez, Egla Marina Gonza'lez Molina, and Jose’ Ramon Padilla Olmedillo. Multiple Bilateral Taurodontism. A Case Report JOE - Vol 36, Number 11, November 2010.

[8] Marques-da-Silva B, Baratto-Filho F, Abuabara A, et al. Multiple taurodontism: the challenge of endodontic treatment. J Oral Sci 2010;52:653-8.

[9] Tsesis I, Shifman A, Kaufman AY. Taurodontism: an endodontic challenge. Report of a case. J Endod 2003;29:353-5.

[10] Demiryeurek Eco, Geoneulol N, Bulucu B. Endodontic treatment of a taurodontic premolar with five canals. Aust Endod J 2013;39:81-4.

[11] Jafarzadeh H, Azarpazhooh A, Mayhall JT. Taurodontism: a review of the condition and endodontic treatment challenges. Int Endod J 2008;41:375-88.

[12] Bharti R, Chandra A, Tikku AP, et al. "Taurodontism” an endodontic challenge:a case report. J Oral Sci 2009;51:471-4. 